\title{
UNBOUNDED OPERATORS COMMUTING WITH RESTRICTED BACKWARD SHIFTS
}

\author{
DONALD SARASON
}

Abstract. The closed densely defined operators on a proper invariant subspace of the backward shift that commute with the restricted backward shift are shown to be coanalytic Toeplitz operators induced by functions in the Nevanlinna class. The result can be interpreted as a kind of commutant lifting theorem for unbounded operators. It extends, and in a certain sense completes, earlier work of Daniel Suárez.

Mathematics subject classification (2000): 47B35, 47A20, 30D50.

Keywords and phrases: Restricted backward shift, commutant lifting, functions of bounded characteristic, Nevanlinna class, Toeplitz operator.

\section{REFERENCES}

[1] H. Helson, Large analytic functions, Linear Operators in Function Spaces (Timişoara, 1988), 209-216, Oper. Theory Adv. Apl. 43, Birkhaüser, Basel, 1990; MR1090128 (92c:30038).

[2] H.Helson, Lectures on Invariant Subspaces, Academic Press, New York and London, 1964; MR0171178 (30\#1409).

[3] N. K. NIKOL'SKII, Treatise on the Shift Operator, Grundlehren der Mathematischen Wissenschaften, 273, Springer-Verlag, Berlin, 1986; MR0827223 (87i:47042).

[4] D. SARASON, Algebraic properties of truncated Toeplitz operators, Oper. Matrices 1 (2007), 491-526; MR2363975.

[5] D. SARASOn, Unbounded Toeplitz operators, Integral Equations Oper. Theory 61 (2008), 281-298.

[6] S. M. SEUBERT, Unbounded dissipative compressed Toeplitz operators, J. Math. Anal. Appl. 290 (2004), 132-146; MR2032231 (2004i:47053).

[7] D. SUÁREZ, Closed commutants of the backward shift operator, Pacific J. Math. 179 (1997), 371-396; MR1452540 (99a:47050).

[8] B. SZ.-NAGY AND C. FOIAŞ, Harmonic analysis of operators on Hilbert space, North-Holland Publishing Co., Amsterdam-London, American Elsevier Publishing Co., Inc., New York, Ahadémiai Kaidó, Budapest, 1970; MR0275190 (43\#947).

[9] R. L. WheEDEn AND A. ZyGMund, Measure and Integral, Marcel Dekker, New York and Basel, 1977; MR0492146 (58\#11295). 